\title{
Online Signature System Based on Pressure and Speed Features
}

\author{
Nor Azlin Rosli and Azlinah Mohamed
}

\begin{abstract}
Online signature or dynamic signature is a biometric modality that uses, for recognition purpose, the behavioural that an individual exhibit when signing. There's a lot of factor that can be dynamically captured in the online signature recognition as the indicator of individual's identity or personality. This research aims to identify the two (2) important features of online signatures, pressure and speed and collaborate with the previous research in HoloCatT Matrix construction by [9]. The process involves data acquisition where the person will sign on the digital tablet and the signature is captured in an $x$ and $y$ values. Subsequently, an algorithm is formulated to capture the preasure and speed of the signature. Both factors have been divided into three (3) categories as reflected in the HoloCatT Matrix formulated in previous research. Five (5) signatures were captured for the functional testing and another six (6) signatures captured for the verification testing. The results from both testing show the accuracy of proposed algorithm and it has been verified by the handwriting expert by comparing the results between online and manual signature analysis.
\end{abstract}

Index Terms-Online signature, HoloCatT matrix, personality.

\section{INTRODUCTION}

Nowadays, there are a lot of applications that have been built for recognition purposes. The process involves any part of our body such as face, eye, thumbprint and etc. Same involves with signature, because most of the selected part that was used in this process is unique, where different people will have different characteristics and ways of presenting it [1]. Based on [2], signature is a behavioral biometric where by a person's signature slightly differ overtime. Therefore signature is not unique or difficult to forge as physical properties. However, the vast usage of signatures in many fields makes it a suitable biometric feature to be used in any authentication applications.

This research will cover an online signature recognition solution with new features that have been identified in previous study in enhancing the HoloCatT Matrix by Nor. et al., and it is an extension from previous research that was done by [3] where they were focusing on five (5) factors of signature recognition which was caliber, proportion, space, baseline, and slanting.

Section II of this paper discusses the related studies on online and offline signature recognition. Meanwhile, section 3 discusses the research methodology followed by the system

Manuscript received July 18, 2012; revised August 30, 2012. This work was supported by the Ministry of Science and Technology Malaysia through the Science Fund.

The authors are with Faculty of Computer Science and Mathematics, Universiti Teknologi MARA, Malaysia. architecture in section 4. Section 5, discusses on the testing procedure and the findings of the research. Finally, the last section concludes the research and future work.

\section{OFFLINE VS. ONLINE SIGNATURE}

An application built based on recognition process could be extended to verify and authenticate applications. The recognition methods are mainly divided into two types, which are the online recognition and offline recognition [4] [5] [6]. In general, through off-line system, data will be captured by scanning an original images into recognition system while on-line system, any data are captured dynamically through a pressure-sensitive device, such as PDAs and Tablet PCs and other pen or paper-based system [7].

\section{A. Offline Signature}

Digitizing a signature from document or paper into a system through intermediate device such as scanner, camera, etc is known as offline signature recognition. As a matter of fact, the description itself shows the overall processes that involve the offline recognition system, which consists of four processes: acquisition, segmentation, recognition and pre-processing [8].

One advantage of offline signature recognition system is that the recognition process can be done at any time after the signature has been made [8]. Besides that, the difference in the pen width and unpredictable change in signature aspect ratio is the challenge in offline recognition system where even professional forensic examiners perform about $70 \%$ of correct signature classification rate (genuine or forgery)[2].

\section{B. Online Signature}

In online handwriting recognition approach, the user writes using a digital device (such as a digital tablet) utilizing a special stylus. In 1980s, "paperlike" interface was introduced, by combining a digitizer and flat display which allows the same surface handling both input and output to provide immediate electronic ink feedback that mimics the paper-and-pen paradigm [8].

Based on the study by [2], online signatures are more unique and difficult to forge than their previous counterparts. In addition, the classification of online signature recognition considers the dynamic signature features like speed, pressure, and time of each point on the signature trajectory. As a matter of fact, since both temporal and spatial information available in the online signature system, this system will produce more accurate result than offline signature system [8].

Furthermore the online signature algorithm takes into account the time and not only the visible ink for recognition or authentication purposes [8]. This obviously would 
increase the reliability of online signature verification as compared to offline.

\section{METHODOLOGY}

This section will elaborate the making of the online signature prototype system in simulating the result for the new version of HoloCatT Matrix in [9].

A waterfall model has been chosen for the system methodology that acts as the backbone that provides guidance on how the prototype system should be developed and the proper activities to be done. The prototype is meant to be a preliminary solution and part of a working process in information system. In the other hand, prototyping itself helps both users and developers where for developers, it may help in assessing the design strategies (design prototype) while for users, this could help them to understand on what will the system look like (user interface prototype) [10].

Fig. 1, illustrates the waterfall model with prototyping together with the activities involve in each stage that represents the system methodology for this research.

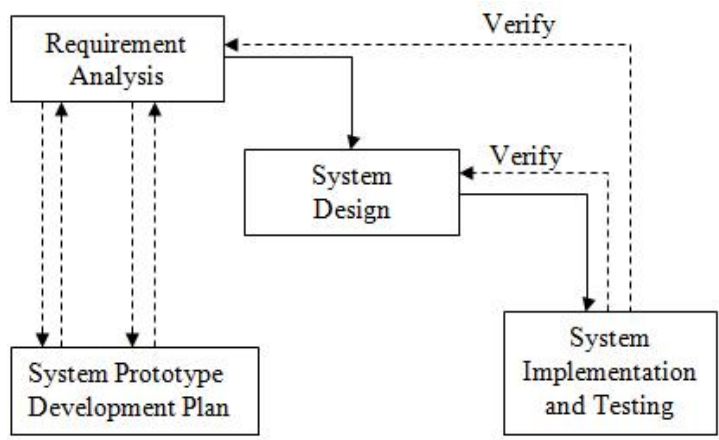

Fig. 1. Waterfall model with prototyping

(Source: Pfleeger, et al., 2006, Software engineering theory and practice)

\section{SYSTEM ARCHITECTURE}

The prototype system comprising hardware and software elements is proposed. Tablet digitizer (Genius Mouse Pen $8 \times 6$ ) with pressure sensitive surface was used in this research to capture absolute pen pressure signal, time and $X Y$ coordinate cooperating with a specific pen. This is the main input device for the data acquisition.

The software architecture of the system involves the use of object oriented programming, and their associated components. Wintab32 API and its library were employed to enable seamless communication. In providing the graphical overview of the prototype system, Fig. 2 shows the flowchart of the prototype system. The view covers the overall processes that are involved in the prototype starting from input process (where user started to sign their signature), the process (calculating and identifying user's pressure and speed) and output being produced (personality of the user being informed).

In reference to the prototype workflow shown in Figure 2, the process starts from the moment users sign their signature on the selected tablet digitizer till it stops identifying any movement. Basic formula of calculation was used in this process. The $\Sigma$ (sum) of pressure was identified based of every single point of pressure $(\mathrm{P})$ that was detected by the sensor pen which has 1024 levels and a range starting from 0 till 1023 divided with $n$, total number of pressure point and as a result an average value obtained will be categorized base on the association Table I below:

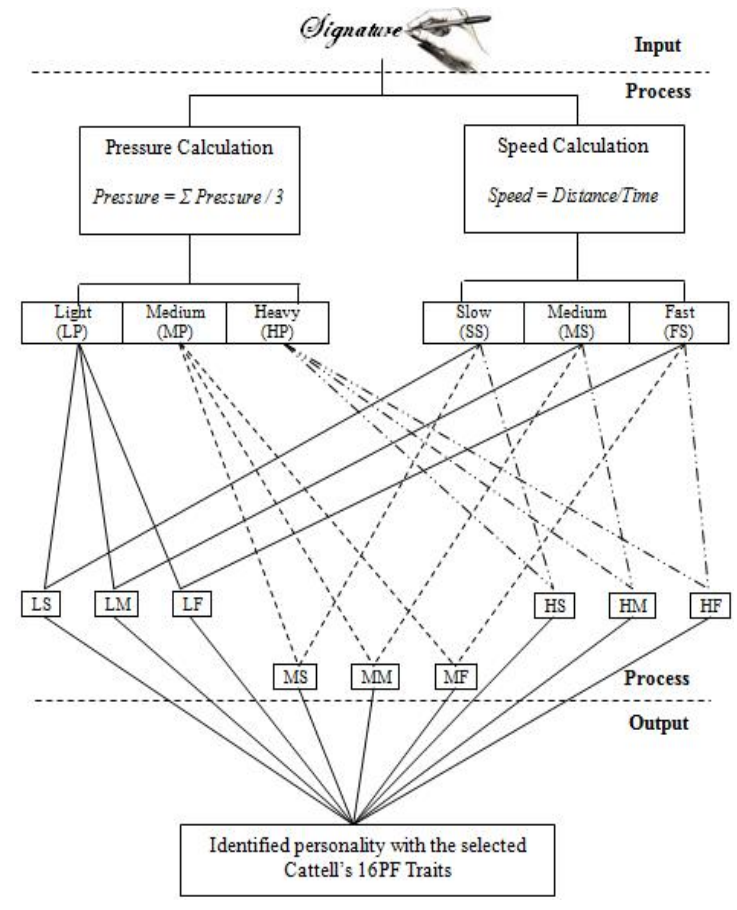

Fig. 2(a) Flowchart of prototype system

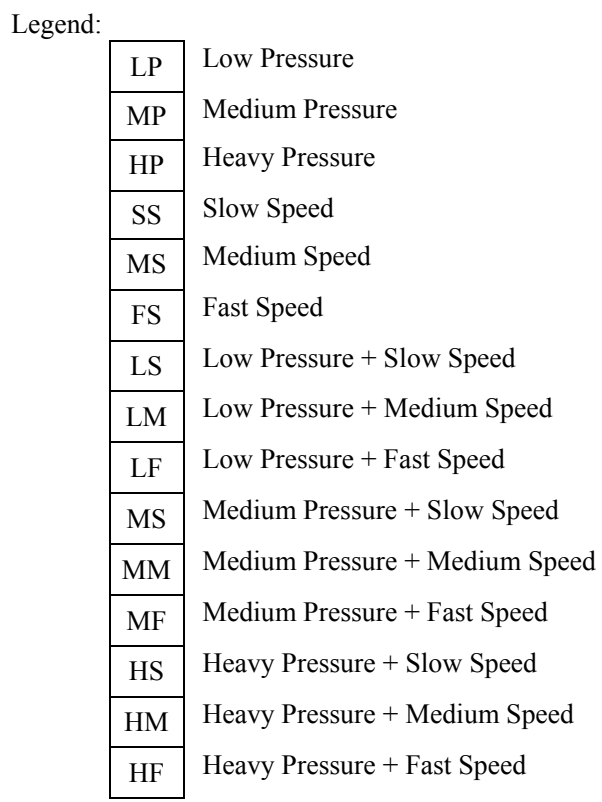

Fig. 2(b) Legend of prototype system flowchart

TABLE I: ASSOCIATION OF VALUE AND STYLES OF PRESSURE

\begin{tabular}{llll}
\hline \hline Value (dpi) & $0-340$ & $341-682$ & $683-1023$ \\
\hline Style & Light Pressure & Medium Pressure & Heavy Pressure \\
\hline
\end{tabular}

Based on the observation and experiment, the digitize pen produced a value of pressure based on every single dot that being put onto the digitize tablet. From the experiment, every dot has a different level of ink where a light ink will be produced when a light pressure was put onto the tablet and the ink will become thicker and thicker when more pressure put on the tablet as illustrated in Fig. 4. 


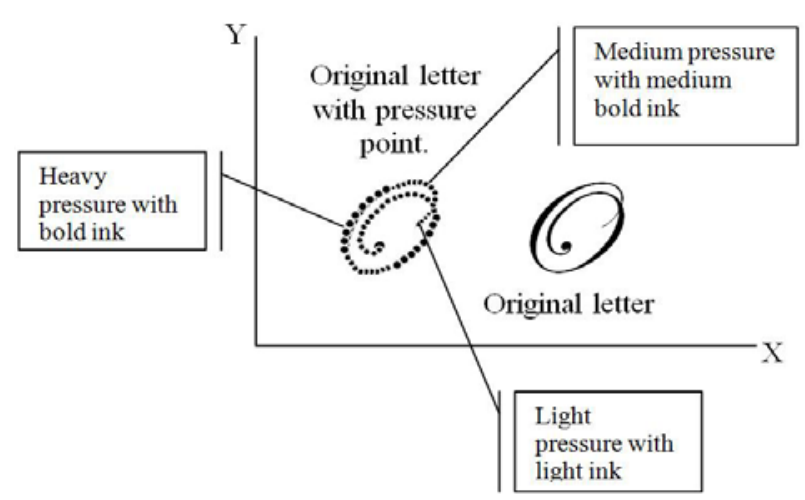

Fig. 4. Sample signature with point of pressure illustrated.

The second calculation of speed of signature, total number of distance which is being identified by getting the difference between coordinate of final stops minus the first dot (Xmax $\left.X_{1}\right)$ and being divided by time taken to perform the signature $(t)$.

$\mathrm{X}$ in the above calculation represents the $\mathrm{X}$ value in the $\mathrm{X}$-axis of the signature that captured from the moment the signature starts till the end of the movement. Fig. 5 shows the sample signature with illustrated $\mathrm{X}$ coordinate.

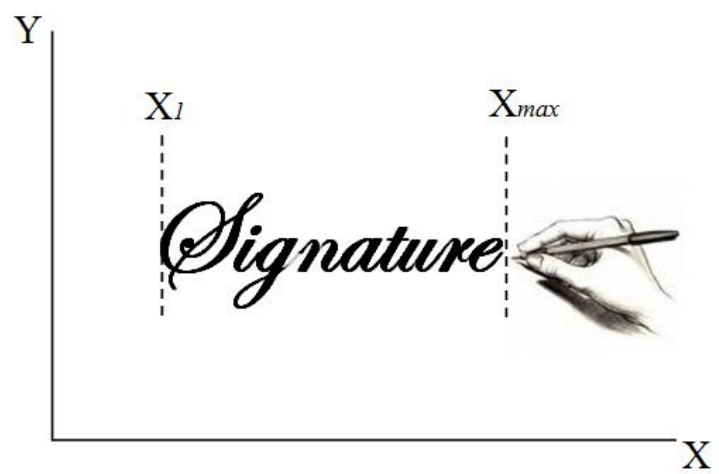

Fig. 5. Sample signature with illustrated $\mathrm{X}$ coordinate

The speed of the signature is categorized into slow, medium and fast speed. A dedicated box with 150 pixel (y-axis) x 300 pixel (x-axis) dimensions was used as an indicator to capture the signature. The categorization of each reading is as shown in Table II.

TABLE II. ASSOCIATION OF VALUE AND STYLES OF SPEED

\begin{tabular}{llll}
\hline \hline Speed $(\mathbf{m} / \mathbf{s})$ & $1-50$ & $51-150$ & $>=151$ \\
\hline Style & Slow Speed & Medium Speed & Fast Speed \\
\hline \hline
\end{tabular}

The fast speed range does not have any maximum value since it depends on how fast a signature was produced in 1 second and based on an interview with the Graphologist, Mr. Darwish Paramjit, $50 \%$ of people will have a fast signature and the percentage will only reduce to $30 \%$ or $20 \%$ base on certain situation such as signing on a check, approving report and etc.

\section{A. Pseudo Code}

In describing the algorithm of the selection process involved in the prototype system, a set of pseudo code was constructed as shown in Fig. 6:

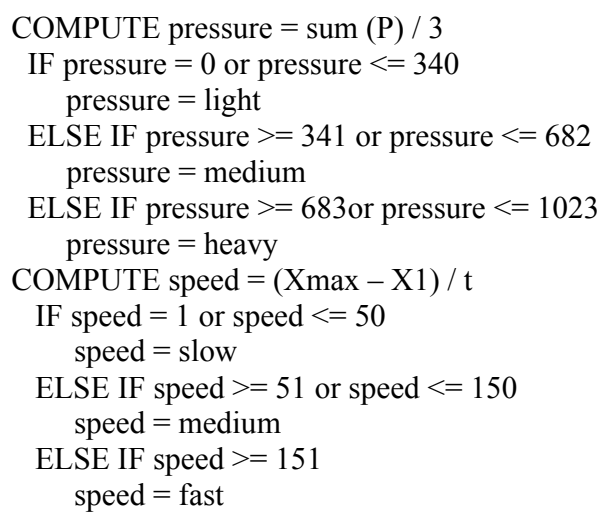

Fig. 6. Pseudo code for identifying the pressure and speed value

Process on identifying the personality based on the 6 identified features which will result in 9 personality possibilities is as illustrated in Figure 7. The personality is determined by taking both pressure and speed features and associate it with the HoloCatT Matrix [3] developed earlier.

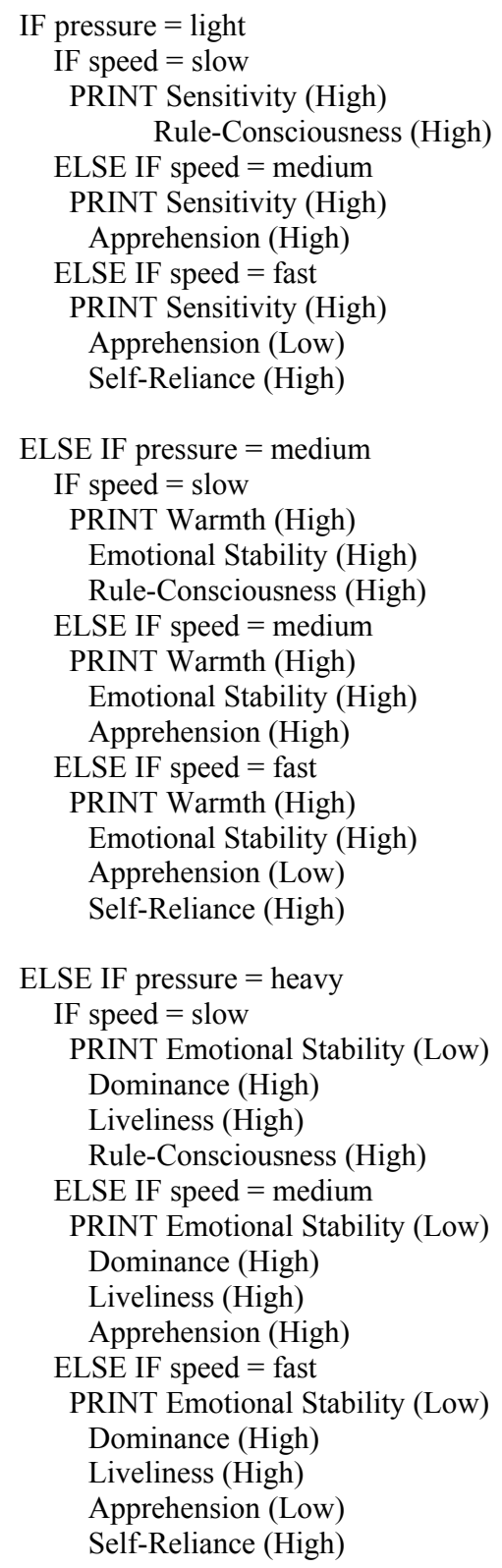

Fig. 7. Pseudo code for personality selection process 


\section{System TeSTING AND FINDINGS}

The results were tested using two (2) types of testing which through functional testing and expert verification testing.

\section{A. Functional Testing}

Functionality testing is employed to verify whether the prototype system meets the intended specifications and functional requirements laid out in the requirement analysis phase. In this phase, 5 signatures have been entered into the prototype to test on the functionality of the system (as shown in Table 3) and the possible outcome based on researcher observations and experiments.

TABLE III: FUNCTIONAL TESTING WITH 5 USERS AND THE RESULTS

\begin{tabular}{ll} 
TABLE III: FUNCTIONAL TESTING WITH 5 USERS AND THE RESULTS \\
\hline Signature & \multicolumn{1}{c}{ Results } \\
\hline Pressure = Light \\
Speed = Slow \\
Personality:
\end{tabular}

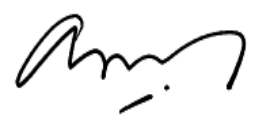

$$
\begin{aligned}
& \text { Pressure = Medium } \\
& \text { Speed = Medium } \\
& \text { Personality: } \\
& \quad \text { Warmth (High) } \\
& \quad \text { Emotional Stability (High) } \\
& \text { Apprehension (High) }
\end{aligned}
$$

Time $=1.986 \mathrm{~s}$

Pressure $=624 \mathrm{dpi}$

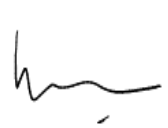

$$
\begin{aligned}
& \text { Pressure = Light } \\
& \text { Speed = Fast } \\
& \text { Personality: }
\end{aligned}
$$$$
\text { Sensitivity (High) }
$$

$\mathrm{Xmax}=199, \mathrm{X}=93$

Time $=0.689 \mathrm{~s}$

Pressure $=292 \mathrm{dpi}$

Apprehension (Low)

Self-Reliance (High)

Based on the functionality testing done in Table 3, it shows that the value of pressure and speed captured by the prototype and the result (personality) produced do have some rational where the results shows that the prototype behaves correctly and logically according to graphologist perspective identified in previous phase and functions which according to the requirements. The testing was supported by direct observation done while user signs onto the digital tablet until they received the results of their personality. In verifying the results based on both usability testing and direct observation, expert verification testing have been performed and discussed in the next phase.

\section{B. Expert Verification}

The verification process involves a graphologist expert to validate the prototype. This phase requires the expert to input his signature 6 times for testing purposes. The result is than compared to the result from a manual signature analysis. Based on the test, expert agrees that the prototype do have similarity with the manual signature analysis except for there is 1 limitation that may cause the signature being produced with different criteria as in real-life environment because they have to concentrate on the screen rather than looking into the tablet directly when signing.

\section{CONCLUSION AND RECOMMENDATION}

This research aims to identify the pressure and speed of a signature collaborates with the previous research in HoloCatT Matrix construction by [9].

Although the research shows a promising result, there are many new techniques that could be adapted for further enhancement for better result such as using fuzzy and hybrid intelligent features that could help to improvise the association table for pressure and speed. It is hoped that this research will contribute some knowledge in signature recognition.

\section{ACKNOWLEDGMENT}

We would like to acknowledge Mr. Dawish Paramjit, Graphologist for his contribution and to those who were involved indirectly in this research.

\section{REFERENCES}

[1] Jain, A K., Griess, F. D. and Connell, S. D., "On-line Signature Verification, Pattern Recognition”, 35, 2963 - 2972, 2002.

[2] Alisher, K., Biometric Identity Verification Using On-Line \& Off-Line Signature Verification, 2003

[3] Mohd, R. S., Ku, S. J., Zaidah, I., Rahmatullah, K. A. W. K., and Azlinah, M. Graphology and Cattell's 16PF Traits Matrix (HoloCatT Matrix), 2007.

[4] Liu, C. L., Jaeger, S., and Nakagawa, M., "On-line recognition of Chinese characters: The state of the art". IEEE Trans. PAMI, 26, pp.198-213, 2004.

[5] Kawamura, A., Yura, K., Hayama, T., Hidai, Y., Minamikawa, T., Tanaka, A, and Masuda, S., On-line recognition of reely handwritten Japanese characters using directional feature densities. Proc. 11th ICPR, 2, pp.183-186, 1992.

[6] Hamanaka, M., Yamada, K., \& Tsukumo, J., On-line Japanese character recognition experiments by an off-line method. Proc. 2nd ICDAR, pp.204-207, 1993.

[7] Hideto O., Bilan, Z., Junko, T, Motoki, O., Akihito, K., and Masaki, N, A Compact On-line and Off-line Combined Recognizer, 2006.

[8] Tappert, C. C., Cha, S.-H.; English Language Handwriting, Recognition Interfaces; In Text Entry Systems: Mobility, Accesibility, Universality; I.S. MacKenzie and K. Tanaka-Ishii (eds); San Francisco: Morgan Kaufmann; pp. 123-138,2007.

[9] Nor, A. R., Azlinah, M., and Rahmattullah, K.,. Visualisation Enhancement of HoloCatT Matrix,2009

[10] Pfleeger, S.L. and Atlee, J.M., Software Engineering: Theory and Practice, Prentice Hall, 2006.

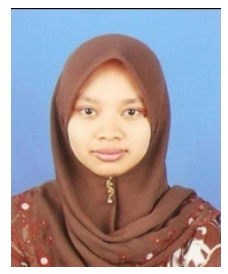

Nor Azlin Rosli is a lecturer in Faculty of Computer Science and Mathematics, Universiti Teknologi MARA, Malaysia. She received her MSc of IT in 2009, BSc of IT in 2005 and Diploma in Computer Science in 2002 from Faculty of Computer Science and Mathematics, Universiti Teknologi MARA, Malaysia. Her research interest was in the decision support system and machine learning.

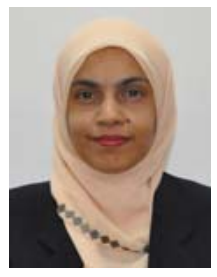

Dr. Azlinah Mohamed is a Professor in Faculty of Computer Science and Mathematics, Universiti Teknologi MARA, Malaysia. She received her Phd in Science and System Management in 2001 and BSc of Computer Science in 1990 from Universiti Kebangsaan Malaysia, MSc in Artificial Intelligence in 1992 from Bristol University, UK. Her research interest was in the artificial intelligence, decision support system, and knowledge base system. 